Supporting Information for

\title{
Amplification of Nuclear Overhauser Effect Signals by Hyperpolarization for Screening of Ligand Binding to Immobilized Target Proteins
}

\author{
Yunyi Wang and Christian Hilty* \\ Chemistry Department, Texas A\&M University, 3255 TAMU, College Station, TX 77843, USA \\ *corresponding author. E-mail: chilty@tamu.edu
}

\section{Table of Contents}

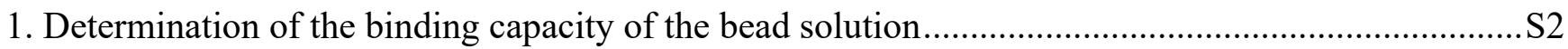

2. Determination of the cross-relaxation rates from conventional NOESY …..................................S3

3. Determination of the dissociation constant for ligand HABA binding to protein avidin ...................S5

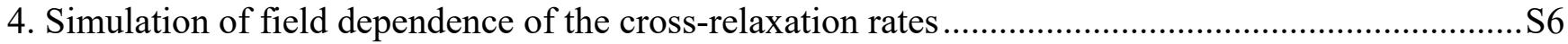

5. Experimental parameters and fitted results of the DNP trNOE experiments ..................................S7

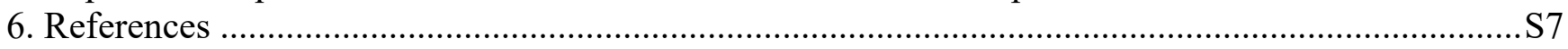




\section{Determination of the binding capacity of the bead solution}

The binding capacity of the concentrated avidin coated bead solution was estimated following a similar procedure described in ref. 1. It was assumed that the bead solution has the same binding capacity for HABA as for the strong ligand biotin. The binding capacity for biotin was determined based on the measurement of the amount of biotin left when removing the biotin-bound bead after adding a certain amount of biotin. $10 \mu \mathrm{L}$ of $40 \mu \mathrm{M}$ biotin solution was added to $30 \mu \mathrm{L}$ of the bead solution, followed by incubation for $5 \mathrm{~min}$ and centrifugation for $5 \mathrm{~min}$. The concentration of biotin left in the supernatant was quantified using the HABA/avidin colorimetric assay. ${ }^{2}$ The supernatant was added to the HABA-avidin solution, causing the displacement of HABA from the complex. The decrease of absorbance of the HABA-avidin complex at $500 \mathrm{~nm}\left(A_{500}\right)$ was measured (Figure S2). A control experiment was performed by replacing the supernatant with $10 \mu \mathrm{M}$ biotin solution. The binding capacity of the bead stock solution was determined as $8.9 \mu \mathrm{M}$.

The manufacturer reported binding capacity is $0.05 \mathrm{nmole}$ biotin per milligram of $0.5 \% \mathrm{w} / \mathrm{v}$ bead suspension in PBS buffer, which corresponds to a bead concentration of $0.25 \mu \mathrm{M}$. The $O D_{600}$ values for the original solution and the concentrated bead solution were measured as 4.13 and 162, respectively. Therefore, the binding capacity of the concentrated bead solution, according to the manufacturer, is 9.8 $\mu \mathrm{M}$, which is within $10 \%$ of the value determined above.

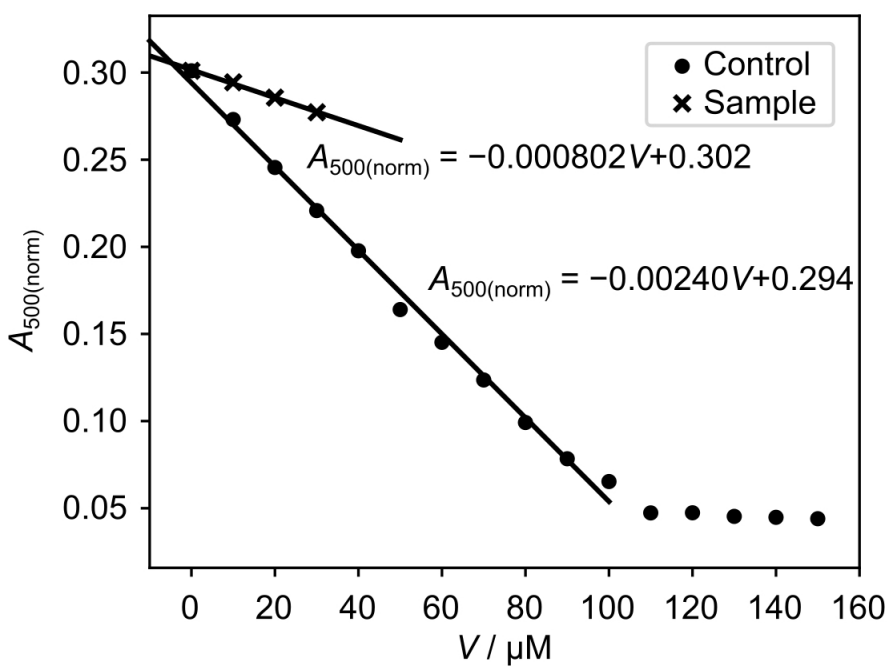

Figure S1. Decrease in absorbance of the HABA-avidin complex at $500 \mathrm{~nm}\left(A_{500}\right)$ when titrating 150 $\mu \mathrm{L}\left(V_{\mathrm{i}}\right) \mathrm{HABA}$-avidin solution with the supernatant from the bead solution and the control biotin solution $(10 \mu \mathrm{M})$, respectively. $V_{\mathrm{a}}$ represents the volume of the sample/control solution added. The absorbances were normalized by the total sample volumes, as $A_{500(\text { norm })}=A_{500} \times\left(V_{\mathrm{a}}+V_{\mathrm{i}}\right) / V_{\mathrm{i}}$. The biotin concentration in the sample supernatant was determined as $3.3 \mu \mathrm{M}$. 


\section{Determination of the cross-relaxation rates from conventional NOESY}

(a)

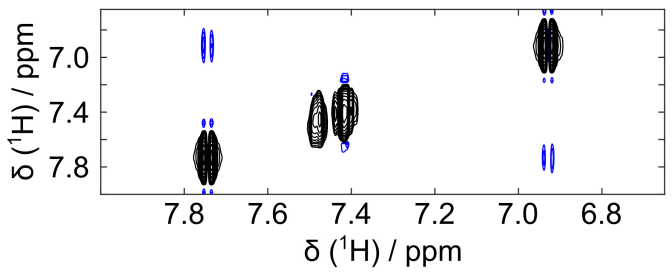

(b)
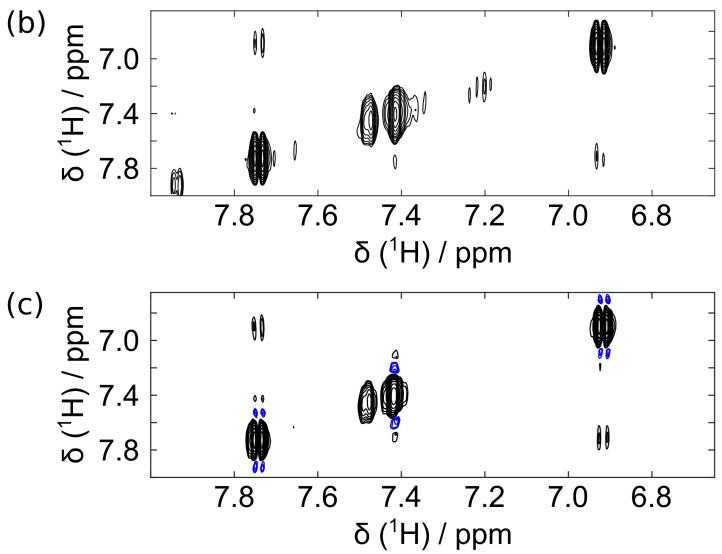

Figure S2. ${ }^{1} \mathrm{H}-{ }^{1} \mathrm{H}$ NOESY spectrum of a) $100 \mu \mathrm{M}$ HABA showing positive NOE b) $100 \mu \mathrm{M}$ HABA with avidin in solution (20 $\mu \mathrm{M}$ binding site) showing negative NOE c) $100 \mu \mathrm{M}$ HABA with avidin immobilized on polystyrene beads $(2.25 \mu \mathrm{M}$ binding site $)$ showing negative NOE. The NOE mixing time was $500 \mathrm{~ms}$, and 128×4096 complex points were collected. 

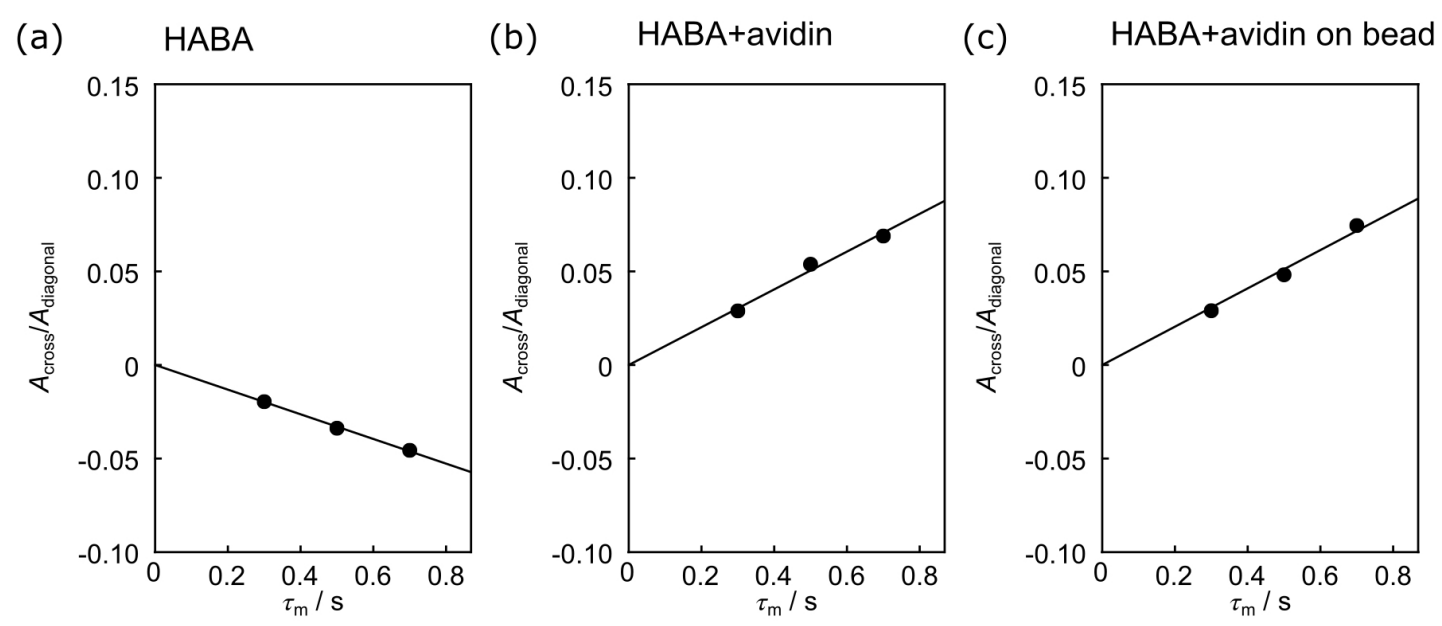

Figure S3. NOE build-up curves in the initial regime measured from ${ }^{1} \mathrm{H}-{ }^{1} \mathrm{H}$ NOESY spectra with mixing times of $300 \mathrm{~ms}, 500 \mathrm{~ms}$, and $700 \mathrm{~ms}$. $A_{\text {cross }}$ represents the average integral of the cross peaks between two $\mathrm{HABA}$ proton spins, $\mathrm{H}_{\mathrm{a}}$ and $\mathrm{H}_{\mathrm{b}}$, while $A_{\text {diagonal }}$ is the average integral of the two diagonal peaks for spin $\mathrm{H}_{\mathrm{a}}$ and spin $\mathrm{H}_{\mathrm{b}}$. Cross-relaxation rates are obtained by a linear fit of $A_{\text {cross }} / A_{\text {diagonal }}\left(\tau_{\text {mix }}\right) .{ }^{3}$ Sample conditions and corresponding fitted cross-relaxation rates are a) $100 \mu \mathrm{M} \mathrm{HABA}, \sigma=0.066 \mathrm{~s}^{-1}$; b) 100 $\mu \mathrm{M}, \mathrm{HABA}$ with avidin $(20 \mu \mathrm{M}$ binding site $), \sigma=-0.101 \mathrm{~s}^{-1}$; c) $100 \mu \mathrm{M}$ HABA with avidin immobilized on polystyrene beads $(2.25 \mu \mathrm{M}$ binding site $), \sigma=-0.102 \mathrm{~s}^{-1}$. 


\section{Determination of the dissociation constant for ligand HABA binding to protein avidin}

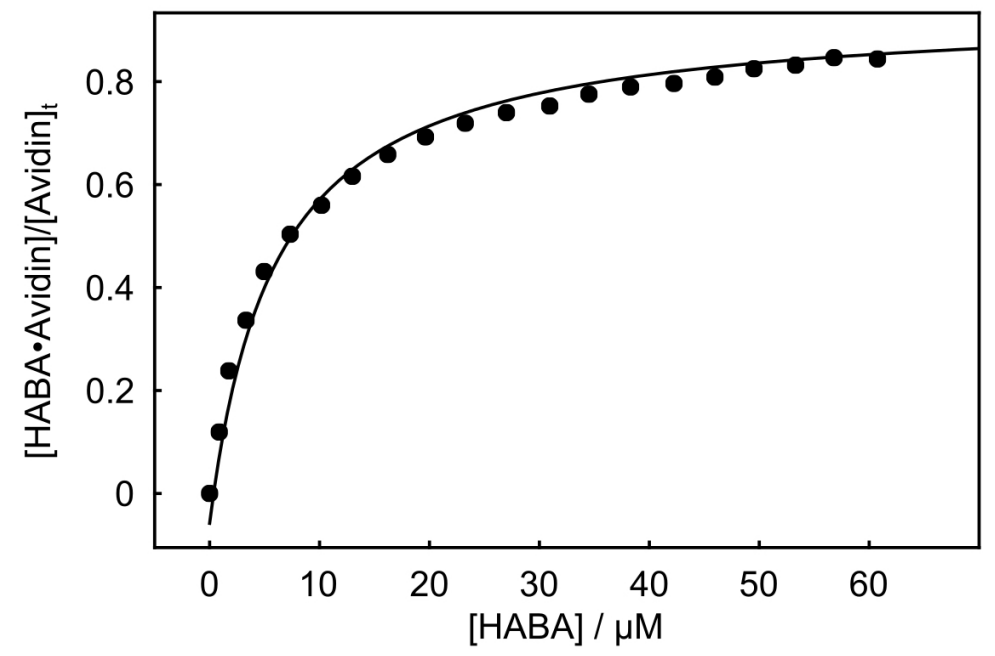

Figure S4. Titration of avidin with HABA. The affinity was determined by measuring the absorbance at $500 \mathrm{~nm}$ using UV/Vis spectrophotometry. A molar extinction coefficient of $35500 \mathrm{~cm}^{-1} \mathrm{M}^{-1}$ for the HABA-avidin complex ${ }^{4}$ was used for calculating the complex concentration [HABA•Avidin]. The fraction of bound protein was fitted with the equation $[\mathrm{HABA} \cdot$ Avidin $] /[\text { Avidin }]_{\mathrm{t}}=$ $[\mathrm{HABA}] /\left([\mathrm{HABA}]+K_{\mathrm{d}}\right)$. The dissociation constant $K_{\mathrm{d}}$ was determined as $5.9 \pm 0.6 \mu \mathrm{M}$, in agreement with the previously reported value of $6 \mu \mathrm{M} .^{5}$ 


\section{Simulation of field dependence of the cross-relaxation rates}

The degree of deviation in the cross-relaxation rates caused by different field strengths can be estimated using the following equations, which take into account only the dipolar relaxation in a two-spin system ${ }^{6}$

$\sigma=\left(\frac{\mu_{0}}{4 \pi}\right)^{2} \frac{\gamma_{\mathrm{H}}^{4} \hbar^{2}}{10} \frac{1}{r^{6}}[(-J(0)+6 J(2 \omega)]$

$J(\omega)=\frac{\tau_{\mathrm{c}}}{1+\left(\omega \tau_{\mathrm{c}}\right)^{2}}$

$\gamma_{\mathrm{H}}$ is the gyromagnetic ratio of the proton, and $r$ is the spin-spin distance. $\sigma$ values at two different field strengths corresponding to ${ }^{1} \mathrm{H}$ frequencies $(\omega)$ of $2 \pi \times 400 \mathrm{MHz}$ and $2 \pi \times 500 \mathrm{MHz}$ were simulated with the molecular correlation time $\left(\tau_{\mathrm{c}}\right)$ changing from $0.01 \mathrm{~ns}$ to $10 \mathrm{~ns}$ (Figure $\mathrm{S} 5$ ).

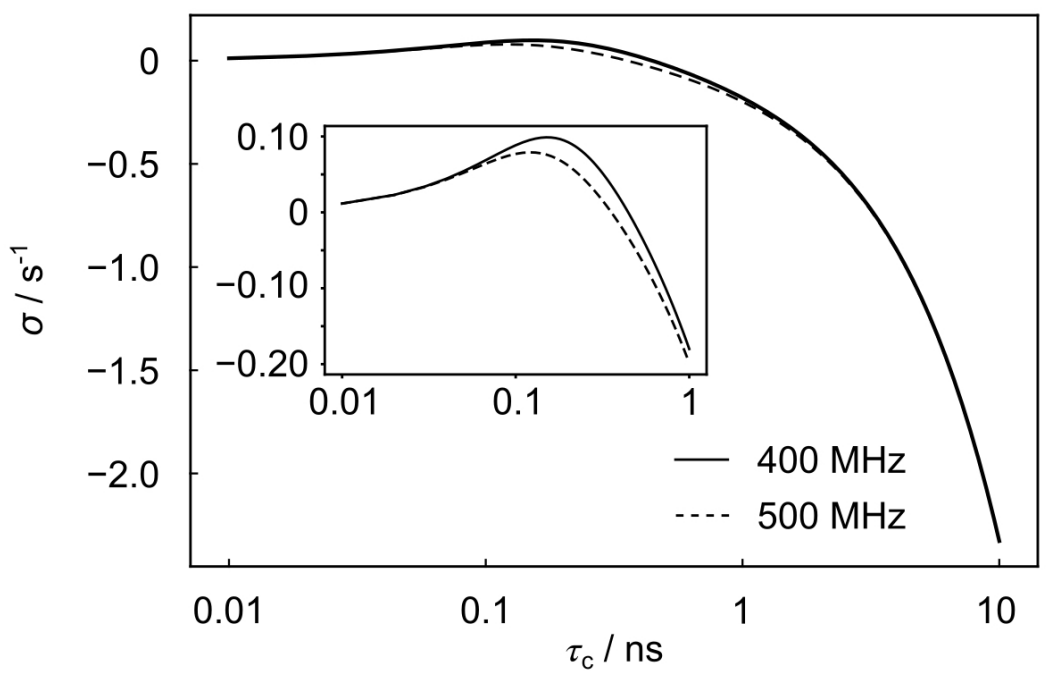

Figure S5. Calculated cross-relaxation rate for a ${ }^{1} \mathrm{H}-{ }^{1} \mathrm{H}$ dipolar spin system at $400 \mathrm{MHz}$ and $500 \mathrm{MHz}$. Eq. (S1) and Eq. (S2) were used to perform the calculations with $r=2.5 \AA$. 


\section{Experimental parameters and fitted results of the DNP trNOE experiments}

Table S1. Experimental parameters and fitted results of the DNP trNOE experiments with hyperpolarized HABA. Exp no. 1-6: HABA only; Exp no. 7-10: HABA mixed with preloaded avidin coated polystyrene particles; Exp no. 11: HABA with preloaded beads and biotin. The enhancement factor $\varepsilon$ for the unsuppressed signal ( $a$ or $b$ ) was determined by comparing the peak integral from the first scan of the DNP experiment with the reference spectrum measured under thermal polarization for the unsuppressed peak. $r_{\mathrm{a}}, r_{\mathrm{b}}$, and $\sigma$ were obtained from fitting the time evolution of peak integrals simultaneously for signal $a$ and $b$ using the Solomon equations (Eq. (1) and Eq. (2) in the text). $\sigma_{\mathrm{b}}$ was determined when immobilized protein is involved, based on the overall cross-relaxation rate $\sigma$ with determined crossrelaxation rate for free ligand $\sigma_{\mathrm{f}}$ and the bound fraction $X_{\mathrm{b}}$ (Formula in Experimental Section).

\begin{tabular}{ccccccccccc} 
Exp no. & $c_{\text {HABA }}(\mu \mathrm{M})$ & $\begin{array}{c}c_{\text {binding site }} \\
(\mu \mathrm{M})\end{array}$ & $X_{\mathrm{b}}$ & $\begin{array}{c}\text { Peak } \\
\text { suppressed }\end{array}$ & $\varepsilon(\mathrm{a}$ or b $)$ & $r_{\mathrm{a}}\left(\mathrm{s}^{-1}\right)$ & $r_{\mathrm{b}}\left(\mathrm{s}^{-1}\right)$ & $\sigma\left(\mathrm{s}^{-1}\right)$ & $\begin{array}{c}\sigma_{\mathrm{b}} \\
\left(\mathrm{s}^{-1}\right)\end{array}$ \\
\hline 1 & 66.9 & - & - & $\mathrm{a}$ & $3.26 \mathrm{E}+03$ & 0.28 & 0.34 & 0.066 & - \\
\hline 2 & 65.7 & - & - & $\mathrm{a}$ & $3.67 \mathrm{E}+03$ & 0.23 & 0.33 & 0.057 & - \\
\hline 3 & 66.6 & - & - & $\mathrm{a}$ & $2.84 \mathrm{E}+03$ & 0.22 & 0.34 & 0.056 & - \\
\hline 4 & 69.7 & - & - & $\mathrm{b}$ & $3.12 \mathrm{E}+03$ & 0.27 & 0.27 & 0.058 & - \\
\hline 5 & 69.7 & - & - & $\mathrm{b}$ & $3.06 \mathrm{E}+03$ & 0.28 & 0.28 & 0.056 & - \\
\hline 6 & 67.6 & - & - & $\mathrm{b}$ & $4.01 \mathrm{E}+03$ & 0.27 & 0.28 & 0.059 & - \\
\hline 7 & 63.5 & 0.83 & 0.012 & $\mathrm{a}$ & $2.11 \mathrm{E}+03$ & 1.00 & 0.75 & -0.055 & -9.5 \\
\hline 8 & 90.4 & 0.83 & 0.009 & $\mathrm{a}$ & $2.59 \mathrm{E}+03$ & 0.87 & 0.67 & -0.032 & -8.5 \\
\hline 9 & 64.7 & 1.15 & 0.016 & $\mathrm{~b}$ & $1.74 \mathrm{E}+03$ & 0.76 & 1.00 & -0.074 & -10.5 \\
\hline 10 & 68.4 & 0.90 & 0.012 & $\mathrm{~b}$ & $2.07 \mathrm{E}+03$ & 0.67 & 0.84 & -0.045 & -8.1 \\
\hline 11 & 58.0 & 0.80 & 0.012 & $\mathrm{~b}$ & $3.13 \mathrm{E}+03$ & 0.39 & 0.37 & 0.017 & - \\
\hline
\end{tabular}

\section{References}

(1) Janolino, V. G.; Fontecha, J.; Swaisgood, H. E. A spectrophotometric assay for biotinbinding sites of immobilized avidin. Appl. Biochem. Biotechnol. 1996, 56 (1), 1-7.

(2) Green, N. M. [74] Spectrophotometric determination of avidin and biotin. Methods Enzymol. 1970, 18, 418-424.

(3) Macur, S.; Farmer, B. T.; Brown, L. R. An improved method for the determination of crossrelaxation rates from NOE data. J. Magn. Reson. 1969 1986, 70 (3), 493-499.

(4) Määttä, J. A. E.; Airenne, T. T.; Nordlund, H. R.; Jänis, J.; Paldanius, T. A.; Vainiotalo, P.; Johnson, M. S.; Kulomaa, M. S.; Hytönen, V. P. Rational modification of ligand-binding preference of avidin by circular permutation and mutagenesis. ChemBioChem 2008, 9 (7), $1124-1135$.

(5) Green, N. M. Avidin. Adv. Protein Chem. 1975, 29, 85-133. 